$03 ; 15$

\title{
Оптоэлектронный двухволновый метод для дистанционного контроля содержания метана в атмосфере
}

\author{
( С.М. Абдурахмонов, О.Х. Кулдашов, И.Т. Тожибоев , Б.Х. Тургунов
}

Ферганский филиал Ташкентского университета информационных технологий им. Мухаммад аль-Харезми, Фергана, Узбекистан

TE-mail: ibroxim@gmail.com

Поступило в Редакцию 14 ноября 2018 г.

В окончательной редакции 14 ноября 2018 г.

Принято к публикации 16 ноября 2018 г.

Предложен оптоэлектронный двухволновый метод и представлена блок-схема оптоэлектронного устройства для дистанционного контроля содержания метана в атмосфере. В оптоэлектронном устройстве в качестве излучающего диода на опорной длине волны использованы светодиоды на основе $\mathrm{GaAlAsSb} / \mathrm{GaInAsSb} / \mathrm{GaAlAsSb}(3.12 \mu \mathrm{m})$, а в качестве излучающего диода на измерительной длине волны светодиоды на основе GaAlAsSb/GaInAsSb/GaAlAsSb $(3.39 \mu \mathrm{m})$.

DOI: 10.21883/PJTF.2019.04.47328.17590

Метан $\left(\mathrm{CH}_{4}\right)$ - один из важных для человечества естественных газов [1]. Концентрация метана формируется в результате биохимических, геохимических и антропогенных процессов. Метан является третьим (после $\mathrm{CO}_{2}$ и $\left.\mathrm{H}_{2} \mathrm{O}\right)$ по важности „парниковым“ газом.

Метан - наиболее важный представитель органических веществ в атмосфере. Увеличение содержания метана в атмосфере способствует усилению парникового эффекта, так как метан интенсивно поглощает тепловое излучение Земли.

Метан в смеси с воздухом горит и взрывается. В зависимости от соотношения концентраций метана и кислорода в воздухе возможны различные виды их реакций при взрыве.

Естественные и антропогенные факторы вызывают необходимость постоянного слежения за ростом и изменениями концентрации приземного метана [2]. Измерения концентрации атмосферного метана проводятся давно. Разработано множество приборов, основанных на различных принципах измерений: диодные лазеры, твердотельные и газовые лазеры, параметрические генераторы, спектрорадиометры и фурье-спектрометры [3].

Задача настоящей работы состоит в создании простого по конструкции устройства - детектора метана, обладающего высокой точностью измерения его концентрации.

Предложен оптоэлектронный двухволновый метод для контроля содержания метана в атмосфере, принцип действия которого заключается в следующем: контролируемый объект облучается двумя противофазными прямоугольными последовательностями импульсов с длинами волн, лежащими в максимуме поглощения контролируемым компонентом (измерительной) и в не максимуме поглощения этим компонентом (опорной).

При этом в случае использования коллимированных потоков излучения достигающий поверхности контроли- руемого объекта поток излучения определяется как

$$
\begin{aligned}
& \Phi_{0 \lambda 1}^{\prime}=\omega_{\lambda 1} \Phi_{0 \lambda 1}, \\
& \Phi_{0 \lambda 2}^{\prime}=\omega_{\lambda 2} \Phi_{0 \lambda 2},
\end{aligned}
$$

где $\omega_{\lambda 1}$ и $\omega_{\lambda 2}-$ коэффициенты пропускания атмосферы на опорных и измерительных длинах волн соответственно.

Отраженные от поверхности объекта контроля потоки излучения на опорных и измерительных длинах волн проходят дистанцию $L$ и воспринимаются одним и тем же приемником излучения. Тогда достигающие поверхности приемника излучений потоки определяются как

$$
\begin{gathered}
\Phi_{\lambda 1}=\gamma_{\lambda 1} I_{0 \lambda 1} \frac{S_{f p}}{L^{2}} e^{-k_{1} N_{1}}, \\
\Phi_{\lambda 2}=\gamma_{\lambda 2} I_{0 \lambda 2} \frac{S_{f p}}{L^{2}} e^{-k_{2} N_{1}} e^{-k_{3} N_{2}},
\end{gathered}
$$

где $\gamma_{\lambda 1}$ и $\gamma_{\lambda 2}-$ коэффициенты отражения объекта контроля на опорных и измерительных длинах волн соответственно; $I_{0 \lambda 1}$ и $I_{0 \lambda 2}$ - интенсивности отраженного от поверхности контролируемого объекта потока излучения на опорных и измерительных длинах волн соответственно; $S_{f p}$ - площадь входного зрачка приемника излучений; $L-$ расстояние, на котором проводится контроль; $k_{1}$ и $k_{2}-$ коэффициенты рассеяния объекта контроля на длинах волн $\lambda_{1}$ и $\lambda_{2}$ соответственно; $N_{1}$ - суммарная концентрация газообразных веществ в объекте контроля; $N_{2}$ - концентрация определяемого газообразного вещества.

В оптоэлектронных устройствах с функциональной разверткой амплитуда одного из потоков излучения (например, измерительного) поддерживается постоянной, а амплитуда другого потока модулируется во времени по экспоненциальному закону. 


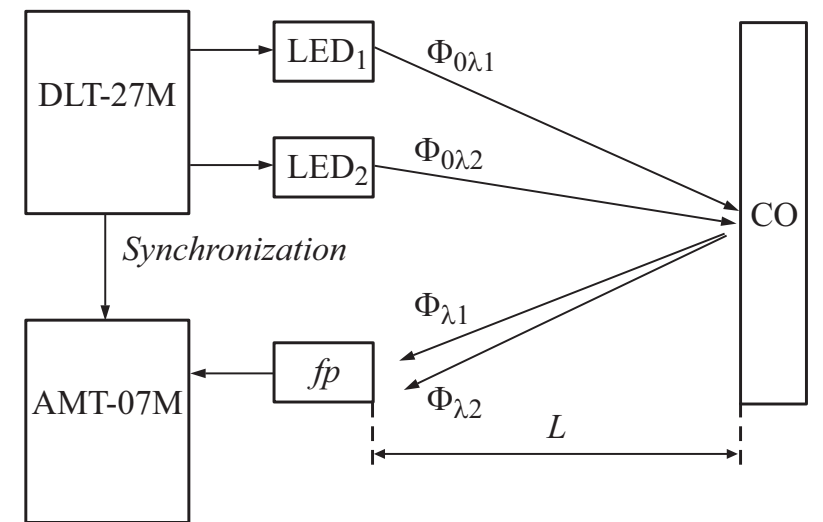

Блок-схема оптоэлектронного устройства для дистанционного контроля содержания метана в атмосфере. DLT-27M источник питания для светодиодов с термохолодильником, AMT-07M - усилитель с термохолодильником, LED 1 светодиод, излучающий на опорной длине волне $(3.12 \mu \mathrm{m})$, $\mathrm{LED}_{2}$ - светодиод, излучающий на измерительной длине волны $(3.39 \mu \mathrm{m}), f p-$ фотодиод серии PD36, CO - контролируемый объект.

Пусть амплитуда опорного потока излучения изменяется по спадающему экспоненциальному закону, т. е.

$$
\Phi_{0 \lambda 1}=A e^{-t / \tau},
$$

где $A$ - амплитуда начального потока излучения на опорных длинах волн; $t$ - текущее время экспоненты; $\tau$ - постоянная времени экспоненты.

Тогда с учетом изложенного выше выражение (5) имеет вид

$$
\Phi_{\lambda 1}=A \gamma_{\lambda 1} I_{0 \lambda 1} \frac{S_{f p}}{L^{2}} e^{-k_{1} N_{1}} e^{-t / \tau}
$$

Приравнивая потоки $\Phi_{\lambda 1}=\Phi_{\lambda 2}$, получим

$$
A \gamma_{\lambda 1} \frac{S_{f p}}{L^{2}} e^{-k_{1} N_{1}} e^{-t_{c o m p} / \tau}=\gamma_{\lambda 2} I_{0 \lambda 2} \frac{S_{f p}}{L^{2}} e^{-k_{2} N_{1}} e^{-k_{3} N_{2}},
$$

где $t_{\text {comp }}$ - момент сравнения потоков $\Phi_{\lambda 1}$ и $\Phi_{\lambda 2}$.

Если длина волны опорного потока излучения выбрана достаточно близкой к длине волны измерительного потока, то можно считать, что $\gamma_{\lambda 1}=\gamma_{\lambda 2}, k_{1}=k_{2}$.

Чтобы получить значение контролируемого параметра, предварительно выравниваем начальные значения потоков. Тогда при условии равных начальных потоков излучения опорного и измерительного потоков имеем

$$
e^{-t_{\text {comp }} / \tau}=e^{-k_{3} N_{2}}
$$

или

$$
N_{2}=\frac{1}{k_{2} \tau} t_{\text {comp }}
$$

Из последнего выражения видно, что концентрация определяемого газообразного вещества пропорциональна времени сравнения $t_{c o m p}$, так как $1 / k_{2} \tau$ является постоянной величиной.
На основе двухволнового метода нами было разработано оптоэлектронное устройство для дистанционного контроля взрывоопасных концентраций углеводородов, скопленных в атмосфере.

Блок-схема оптоэлектронного устройства для дистанционного контроля концентрации метана в атмосфере приведена на рисунке.

В оптоэлектронном устройстве в качестве излучающего диода на опорной длине волны использованы светодиоды на основе GaAlAsSb/GaInAsSb/GaAlAsSb $(3.12 \mu \mathrm{m})$, а в качестве излучающего диода на измерительной длине волны - светодиоды на основе $\mathrm{GaAlAsSb/GaInAsSb/GaAlAsSb}(3.39 \mu \mathrm{m})$ [4], которые имели следующие преимущества:

- ширина спектра светодиодов $(0.5 \mu \mathrm{m})$ сравнима с шириной полос поглощения газов, поэтому нет необходимости в использовании дополнительных фильтров;

- существенно более низкая потребляемая электрическая мощность;

— время жизни 80000-100000h непрерывной работы (более чем на порядок превышает время жизни тепловых источников).

\section{Список литературы}

[1] Karol I.L. The methane cycle // NATO ASI Ser. 1993. V. 18. $153 \mathrm{p}$.

[2] Werle P., Slemr F., Maurer K., Kormann R., Mücke R., Jänker B. // Opt. Laser. Eng. 2002. V. 37. N 2-3. P. 101-114.

[3] Richter D., Lancaster D.G., Tittel F.K. // Appl. Opt. 2000. V. 39. N 24. P. 4444-4450.

[4] Журтанов Б.Е., Иванов Э.В., Именков А.Н., Колчанова Н.М., Розов А.Е., Стоянов Н.Д., Яковлев Ю.П. // Письма в ЖТФ. 2001. Т. 27. В. 5. С. 1-7. 